\title{
Atendimento odontológico à criança com Síndrome de Down: Revisão da literatura
}

\author{
Dental care for children with Down Syndrome: Literature review \\ Atención dental para niños con Síndrome de Down: Revisión de la literatura
}

Recebido: 31/10/2021 | Revisado: 04/11/2021 | Aceito: 08/11/2021 | Publicado: 13/11/2021

\author{
Terezinha Álvares de Deus Neta \\ ORCID: https://orcid.org/0000-0002-6406-4594 \\ Centro Universitário de Patos de Minas, Brasil \\ E-mail: terezinhadeus@unipam.edu.br \\ Carolina Silva Pereira \\ ORCID: https://orcid.org/0000-0003-2020-3932 \\ Centro Universitário de Patos de Minas, Brasil \\ E-mail: carolinasp@unipam.edu.br \\ Dayviddy Lucas Magalhães Silva \\ ORCID: https://orcid.org/0000-0002-6708-2659 \\ Centro Universitário de Patos de Minas, Brasil \\ E-mail: dayviddylms@unipam.edu.br \\ Laura Cesário Oliveira \\ ORCID: https://orcid.org/0000-0002-7689-5061 \\ Centro Universitário de Patos de Minas, Brasil \\ E-mail: lauracesario@unipam.edu.br \\ Aletheia Moraes Rocha \\ ORCID: https://orcid.org/0000-0003-1650-7546 \\ Centro Universitário de Patos de Minas, Brasil \\ E-mail: aletheiamoraes@unipam.edu.br \\ Daniela Navarro Ribeiro Teixeira \\ ORCID: https://orcid.org/0000-0001-5485-8107 \\ Centro Universitário de Patos de Minas, Brasil \\ E-mail: danielanrteixeira@unipam.edu.br \\ Fabrício Campos Machado \\ ORCID: https://orcid.org/0000-0003-4603-8795 \\ Centro Universitário de Patos de Minas, Brasil \\ E-mail: fabriciocampos@unipam.edu.br
}

\begin{abstract}
Resumo
A síndrome de Down (SD) é um distúrbio cromossômico autossômico causado pela trissomia do cromossomo 21 . É a anomalia mental congênita mais frequente e abrange inúmeras alterações mentais e comportamentais incluindo alterações físicas e orais, as quais necessitam atenção por parte dos cirurgiões dentistas. O tratamento odontológico desses pacientes consiste em eliminar ou controlar as dificuldades que eles possuem, adequando-se a conduta e fazendo com que a abordagem precoce seja a melhor opção. O cirurgião dentista, quando ciente de todas essas complicações e características intra e extra orais, poderá realizar um melhor manejo odontológico. O presente trabalho trata-se de uma revisão narrativa qualitativa, realizada nas bases de dados Biblioteca Virtual em Saúde (BVS), Pubmed e Scielo, utilizando as palavras-chave: "Síndrome de Down", "odontologia" e "manejo comportamental", bem como seus correspondentes em inglês. O trabalho tem como objetivo caracterizar as técnicas de gerenciamento comportamental utilizadas no atendimento odontopediátrico de crianças portadoras da Síndrome de Down e identificar os principais pontos acerca da conduta do cirurgião dentista durante atendimento odontológicos destes pacientes. Com esse estudo, concluiu-se que ainda há bastante controvérsias entre os autores no que se diz a respeito ao alto ou baixo índice de lesão de cárie desses pacientes. Entende-se também, a importância do acompanhamento familiar durante todo o tratamento odontológico e a necessidade do cirurgião dentista de estar bem preparado e saber lidar com todas as características e particularidades de cada criança.
\end{abstract}

Palavras-chave: Síndrome de Down; Odontologia; Manejo comportamental.

\begin{abstract}
Down syndrome (DS) is an autosomal chromosomal disorder caused by trisomy 21. It is the most frequent congenital mental anomaly and encompasses numerous mental and behavioral changes, including physical and oral changes, which require attention from dental surgeons. The dental treatment of these patients consists in eliminating or controlling the difficulties they have, adapting the conduct and making an early approach as the best option. The dental surgeon, when aware of all these complications and intra and extra oral characteristics, will be able to perform a better dental management. The present work is a qualitative narrative review, carried out in the Virtual Health Library (VHL),
\end{abstract}


Pubmed and Scielo databases, using the keywords: "Down syndrome", "dentistry" and "behavioral management", as well as their English counterparts. The study aims to characterize the behavioral management techniques used in pediatric dental care for children with Down syndrome and to identify the main points about the conduct of the dental surgeon during dental care for these patients. With this study, it was concluded that there is still considerable controversy among authors regarding the high or low rate of caries lesions in these patients. It is also understood the importance of family monitoring throughout the dental treatment and the need for the dental surgeon to be well prepared and know how to deal with all the characteristics and particularities of each child.

Keywords: Down syndrome; Dentistry; Behavioral management.

\section{Resumen}

El síndrome de Down (SD) es un trastorno cromosómico autosómico causado por la trisomía 21. Es la anomalía mental congénita más frecuente y engloba numerosos cambios mentales y de comportamiento, incluidos cambios físicos y orales, que requieren la atención de los cirujanos dentistas. El tratamiento odontológico de estos pacientes consiste en eliminar o controlar las dificultades que tienen, adaptando la conducta y haciendo de un abordaje temprano la mejor opción. El cirujano dentista, al conocer todas estas complicaciones y características intra y extraorales, podrá realizar un mejor manejo odontológico. El presente trabajo es una revisión narrativa cualitativa, realizada en las bases de datos de la Biblioteca Virtual en Salud (BVS), Pubmed y Scielo, utilizando las palabras clave: "síndrome de Down", "odontología" y "manejo conductual", así como sus contrapartes en inglés. El trabajo tiene como objetivo caracterizar las técnicas de manejo conductual utilizadas en la atención odontológica pediátrica para niños con síndrome de Down e identificar los principales puntos sobre la conducta del cirujano dentista durante la atención odontológica de estos pacientes. Con este estudio se concluyó que aún existe una considerable controversia entre los autores en cuanto a la alta o baja tasa de lesiones cariosas en estos pacientes. También se comprende la importancia del seguimiento familiar durante todo el tratamiento odontológico y la necesidad de que el cirujano dental esté bien preparado y sepa lidiar con todas las características y particularidades de cada niño.

Palabras clave: Síndrome de Down; Odontología; Manejo del comportamiento.

\section{Introdução}

A Síndrome de Down (SD) é uma anomalia que afeta todos os sistemas do corpo, sendo descrita inicialmente por Langdon Down, em 1866, como um quadro clínico com identidade própria. É a anomalia congênita mais frequente, com maior prevalência e afeta todas as raças e níveis socioeconômicos, englobando alterações mentais e comportamentais além de malformações físicas e orais (Moreira et al., 2015; Pini et al., 2016). O paciente com SD é considerado especial, e deve-se entender que o conceito de paciente especial é definido como todo indivíduo, adulto ou criança que se desvia fisicamente, socialmente ou emocionalmente do que é considerado padrão no que tange ao crescimento e desenvolvimento, requerendo instrução suplementar, educação especial e condutas adequadas por toda sua vida (Pini et al., 2016).

É importante ressaltar que não se trata de uma doença, mas uma condição do indivíduo, onde não se fala em cura ou tratamento, mas sim no controle das condições decorrentes dessa anomalia, apresentadas de forma sistêmica ou local. Crianças que vivem com a síndrome são menores e seu desenvolvimento físico, mental e intelectual pode ser mais lento do que o de outras crianças da sua idade (Vilela et al., 2018). Estima-se que no Brasil ocorra um caso em cada 700 nascimentos, totalizando aproximadamente 270 mil pessoas com Síndrome de Down e no mundo, a incidência estimada é de 1 em 1 mil nascidos vivos (Brasil., 2020).

Em odontologia, pacientes com essa síndrome necessitam de um atendimento individualizado e diferenciado, visto que são considerados especiais e apresentam importantes alterações bucais como: alterações oclusais, alterações na tonicidade da musculatura, ressecamento de mucosa oral, bruxismo, macroglossia, dentre outros (Camera et al.,2011). Os mesmos podem ainda apresentar dificuldade de manter uma higiene oral adequada, além de respiração bucal e dieta cariogênica, o que explica a alta incidência da doença cárie e gengivite (Pini et al., 2016). Entretanto, alguns autores ainda consideram controversos os estudos acerca de cárie dentária em indivíduos com síndrome de Down (Moreira et al., 2015).

A emoção e o comportamento de uma criança durante o tratamento odontológico configuram uma ampla preocupação para os odontopediatras e cientistas (Khandelwal et al., 2019). O comportamento de medo e não colaborativo, como choro, gritos e movimentos corporais pode dificultar e atrasar a qualidade do atendimento odontológico. Se esse problema não for solucionado 
adequadamente, uma resposta negativa persistente é possível de acontecer, sendo um obstáculo para o atendimento odontológico de costume (Khandelwal et al., 2019). Dito isso, os cirurgiões dentistas, em especial os odontopediatras e sua equipe, devem estar aptos a realizarem técnicas de manejo comportamental, preparados para possíveis situações que gerem ansiedade, medo ou demais comportamentos negativos apresentados por esses pacientes (Albuquerque et al., 2010).

A não colaboração do paciente deve-se geralmente a fatores como: traumas passados, ansiedade e condições fisiológicas (Brandenburg \& Marinho-Casanova., 2013). Cabe ao profissional da odontologia a habilidade para lidar e entender as particularidades de cada criança, com o pensamento de que cada uma tem sua história, seus costumes e suas dificuldades (Moura et al., 2015). No contexto das necessidades especiais, como a Síndrome de Down, torna-se ainda mais necessário uma anamnese detalhada e cooperação multiprofissional (Moura et al., 2015). É imprescindível também que haja um preparo da criança por parte dos pais ou responsáveis, buscando sempre ferramentas positivas que minimizem a incerteza em relação ao tratamento odontológico (Moura et al., 2015).

Observando que o paciente com SD é um paciente especial, e que a conduta acerca de seu atendimento deve ser individualizada e diferenciada, verifica-se que o mesmo ainda apresenta diversas modificações orais e alterações que competem a atenção do cirurgião dentista. Dito isso, o objetivo principal deste estudo foi caracterizar as técnicas de gerenciamento comportamental utilizadas no atendimento odontopediátrico de crianças com Síndrome de Down e identificar os principais pontos acerca da conduta do cirurgião dentista durante atendimento odontológicos destes pacientes, incluindo a descrição das principais alterações/condições e de um panorama geral da síndrome em odontologia.

\section{Metodologia}

O presente trabalho trata-se de uma revisão narrativa da literatura, de caráter qualitativo, feito através de pesquisa dos artigos nas plataformas Biblioteca Virtual em Saúde (BVS), Pubmed e Scielo entre os meses de fevereiro e junho de 2021, com a intenção de mapear o conhecimento sobre a questão explicitada (Cordeiro et al., 2007). Os termos designados para pesquisa foram "Síndrome de Down", "odontologia" e "manejo comportamental", bem como seus correspondentes em inglês, sendo os termos utilizados indexados aos descritores de ciência e saúde (DeCS). Os critérios de inclusão foram artigos da língua portuguesa e inglesa, publicados nos últimos 10 anos, com exclusão de artigos de opinião, editoriais e resumos, ainda que publicados em periódicos indexados. Para a seleção dos estudos foi necessário identificar os artigos que se relacionavam ao objetivo principal, realizando a leitura dos títulos, dos resumos e dos artigos. Após análise crítica dos estudos, os mesmos foram sintetizados e agrupados em temas pertinentes a temática principal do estudo, permitindo observar, classificar e descrever os dados adquiridos.

\section{Resultados e Discussão}

\subsection{Contextualização da Síndrome de Down na odontologia}

Pacientes com necessidades especiais são aqueles indivíduos que possuem limitações físicas, mentais e sociais, interferindo diretamente no comportamento e desenvolvimento deles dentro da sociedade e dentro do consultório odontológico, necessitando de um atendimento diferenciado e de máxima atenção (Falcão et al., 2019).

A síndrome de Down (SD) é um distúrbio cromossômico autossômico causado pela trissomia do cromossomo 21, reconhecida também como Trissomia 21, Trissomia G ou Mongolismo (Al-Maweri \& Al-Sufyani., 2014). A mesma é clinicamente caracterizada por baixa estatura; braquicefalia; malformação dos pavimentos auriculares; fendas palpebrais oblíquas; base nasal achatada; cabelos finos, sedosos e ralos; membros curtos com prega palmar transversal; anomalias dentárias e dismorfologia orofacial, necessitando de uma atenção significativa no tratamento odontológico desses pacientes (Al-Maweri 
\& Al-Sufyani., 2014).

Dentre as doenças crônicas sistêmicas apresentadas pelo paciente com SD, podemos citar as cardiopatias, onde a mais comum é o prolapso da válvula mitral, havendo probabilidade 3 a 8 vezes maior de ocorrer quadros de endocardite bacteriana, sendo sugerido que o dentista faça uma profilaxia antibiótica antes de um procedimento invasivo (Al-Maweri \& Al-Sufyani., 2014; Falcão et al., 2019). Outra condição frequente nesses pacientes é o hipotireoidismo, uma disfunção na tireoide, em que o desenvolvimento dos ossos e dentes é afetado, causando um atraso na erupção tanto dos dentes decíduos quanto dos permanentes. Também é comum ocorrerem alterações neurológicas e nas vias respiratórias, com predominância na garganta, ouvido e vias áreas por consequência da deficiência imune, hipotonia muscular e anatomia alterada, possibilitando um maior acúmulo de bactérias, sobretudo nos primeiros anos de vida (Falcão et al., 2019).

Pessoas com SD possuem características orofaciais importantes e um risco maior de desenvolverem problemas de saúde bucal. O processo de desenvolvimento normal dessas estruturas pode ser frequentemente alterado como, por exemplo, menor tamanho dos dentes, erupção atrasada, agenesia e alteração na anatomia da coroa. A função também pode ser comprometida limitando o desenvolvimento da fala, mastigação, deglutição e sucção (Vilela et al., 2018). Também possuem uma maior predisposição a distúrbios orofaciais tais como doença periodontal, má oclusão, distúrbios dos tecidos moles, incluindo protrusão da língua ou lábios invertidos. Os sintomas orais como dor, desconforto ou dificuldade de mastigar, e os problemas sistêmicos, nutritivos e digestivos podem causar grande impacto na vida desses indivíduos, estendendo-se a efeitos maiores na qualidade de vida, entre eles sociais e emocionais (Aljameel et al., 2020).

Em um estudo realizado por Santos et al. (2014), mostrou-se que a giroversão, a agenesia e dentes conóides foram as anomalias de desenvolvimento mais comuns em pacientes com síndrome de Down. Também foram frequentes a presença de dentes supranumerários, atraso da dentição permanente decorrente da retenção prolongada dos decíduos, macroglossia, taurodontia, maxila atrésica, microdontia, hipotonia muscular da língua, oligodontia, bruxismo, queilite angular hipodontia, língua fissurada, respiração bucal, defeito em esmalte, e problemas periodontais (Nacamura et al., 2015; Usui et al., 2020).

A literatura ainda é bem controversa quando se fala de doença cárie nesses pacientes. Pini et al. (2016) e Ghaith et al. (2019) defendem a ideia de que eles possuem uma alta incidência de cárie pelo fato de não realizarem a higienização correta, falta de orientação profissional e devido a algumas limitações motoras e mentais. Já de acordo com Scalioni et al. (2017), esses pacientes possuem um baixo índice de desenvolver lesões de cárie por apresentar uma maior tendência ao bruxismo, desgastando assim as oclusais dos dentes e os deixando sem sulcos e retenções, evitando o acúmulo de alimentos e consequentemente a instalação da doença. Silva \& Trabaquin (2019) defendem também que seja pela maior capacidade tampão da saliva e pelo fato desses pacientes possuírem espaços interproximais decorrentes da agenesia, dificultando assim o acúmulo de restos alimentares e bactérias cariogênicas posteriormente.

A erupção dentária em crianças com a síndrome pode demorar até o $12^{\circ}$ mês, enquanto o molar permanente começa a apontar por volta dos 8 anos de idade. O prognatismo mandibular também é muito frequente, causando a obstrução da oclusão e a mordida invertida, aberta ou cruzada, uni ou bilateral. A macroglossia pode ser real ou aparente em razão da cavidade oral ser menor, e como consequência o paciente adquire hábito de respiração oral e vazamento salivar. A gengivite também é muito comum, por isso a importância de uma higiene bucal diária e controle odontológico de rotina (Gallo et al., 2019).

O desenvolvimento oral infantil é considerado entre zero e um ano e meio de vida. Nesse período, a boca é a estrutura sensorial mais desenvolvida e que provoca maior prazer na criança, e é nessa fase que a criança se adapta ao meio ambiente e entra em contato com o mundo. Pela boca ela se comunica, se alimenta e respira. Diante dessas importantes transformações de crescimento e desenvolvimento, a abordagem do odontopediatra se torna muito conveniente (Falcão et al., 2019). 


\subsection{Atendimento odontológico}

De acordo com a Organização Mundial de Saúde (OMS), 10\% da população mundial possuem algum tipo de deficiência e só 3\% recebem atendimento apropriado. Atualmente, a procura por profissionais da odontologia que realizam um atendimento adequado em pacientes com necessidades especiais, inclusive da SD, está cada vez maior, ao mesmo tempo, porém, em que o despreparo e o desconhecimento por parte desses profissionais é grande (Brasil, 2019).

$\mathrm{O}$ tratamento odontológico consiste em eliminar e/ou controlar as dificuldades que esses pacientes possuem por conta de suas limitações. Eles devem receber uma atenção redobrada no tratamento periodontal, nas lesões de cárie, má oclusão, e apneia obstrutiva do sono. E, por mais que vários métodos existam para melhorar sua higiene bucal, apenas as manobras mecânicas não são suficientes. As técnicas utilizadas no manejo odontopediátrico de pacientes com SD apresentam vários benefícios, podendo evitar o agravamento das doenças bucais (Morales-Chávez et al., 2014).

Os cirurgiões dentistas em alguns casos escolhem a anestesia geral pela grande dificuldade em proceder com tratamentos mais invasivos como a cirurgia, facilitando a reabilitação oral e o manejo com o paciente em uma única sessão. Porém, essa opção é indicada somente quando todos os outros métodos tiverem sido ineficientes (Usui et al., 2020).

A abordagem precoce é sempre a melhor opção. As técnicas de manejo em odontopediatria, juntamente com ajuda dos pais, vão proporcionar a essa criança um vínculo entre profissional e paciente, gerando conforto e confiança mesmo com sua deficiência neurológica, o que facilita o trabalho do cirurgião-dentista. As orientações de como cuidar da alimentação, tanto para evitar obesidade como para saúde oral, e a demonstração do método de higienização da boca, devem ser repassadas com o apoio da família, criando hábitos precoce e acompanhamento de profissionais adequados e habilitados (Morales-Chávez et al., 2014).

Nota-se que a ansiedade e a resposta emocional de cada criança é o que mais dificulta na hora do atendimento odontológico e consequentemente os pais hesitam em levar os filhos, gerando tratamentos mais caros e complexos no futuro (Khandelwal et al., 2019). Segundo Brandenburg \& Marinho-Casanova (2013), os pais têm um papel fundamental na consulta odontológica de seus filhos. A partir do momento em que se tem a cooperação entre os responsáveis, não somente no consultório como também no ambiente familiar, facilita-se a colaboração da criança para o tratamento odontológico, aumento assim as taxas de sucesso.

Para melhor atender o paciente com SD, o profissional da odontologia deve estar ciente de todas as suas individualidades. Uma anamnese bem feita, o uso de um reforço positivo, atenção nas expressões, gestos, reações, atendimento pontual, consultas curtas, e de preferência procedimentos mais simples na primeira sessão, são cuidados essenciais que devem ser priorizados (Falcão et al., 2019).

\subsection{Técnicas de gerenciamento comportamental para pacientes com Síndrome de Down}

A partir do comportamento apresentado pela criança no atendimento odontológico, o profissional pode utilizar recursos para gerenciar o comportamento desse paciente de forma lúdica, fazendo com que o atendimento seja viabilizado, a interação profissional-paciente não seja traumática e tenha segurança e tranquilidade. Dentre as técnicas utilizadas, as principais para pacientes com SD são: comunicação verbal e não verbal, dizer-mostrar-fazer, controle de voz, reforço positivo, distração e dessensibilização, ludoterapia, e caso necessário e pré-estabelecido com os responsáveis, estabilização protetora (Caldas Jr \& Machiavelli, 2013; Silva et al., 2016).

$\mathrm{Na}$ comunicação verbal, expressa-se os procedimentos ao paciente, explicando o que será realizado em seu tratamento, enquanto na comunicação não-verbal observa-se o contato, postura, expressão facial e corporal, reforçando o que foi passado pela primeira (Dias, 2013; Silva et al., 2016).

A técnica do dizer-mostrar-fazer envolve a explicação de forma simples e adequada para a idade e condição do paciente (dizer), demonstrar em um modelo visual e tátil (mostrar) tranquilizando-o, e realizar o procedimento no próprio paciente (fazer). 
Indica-se começar o atendimento sem auxílio de instrumento e ao longo do tempo, com explicações, a criança visualiza e acompanha o processo. É umas das técnicas mais utilizadas, indicada para todos os tipos de pacientes, onde eles se sentem à vontade e criam um vínculo de confiança (Sant’anna et al., 2020).

No controle de voz, o volume e o tom desta vão se adaptando de acordo com a necessidade, para direcionar ou influenciar o comportamento desejado. A entonação e a expressão facial do profissional são fatores importantes que contribuem e refletem a atitude de confiança e controle. Dessa forma, além de oferecer a orientação à criança, haverá também a cooperação da mesma. É contraindicada apenas para os pacientes com deficiência auditiva (Caldas Jr \& Machiavelli, 2013; Silva et al., 2016).

O reforço positivo se caracteriza como o processo de motivar a criança com elogios, expressão facial positiva, gestos positivos e lembrancinhas. Em caso de comportamentos negativos, as palavras de ordem "pare" e "não faça" devem ser evitadas para não ameaçar e prejudicar a cooperação do paciente, é importante o profissional manter a calma e pedir gentilmente sua cooperação para o que está sendo proposto, podendo esta técnica ser usada para todos os pacientes (Caldas Jr \& Machiavelli, 2013; Silva et al., 2016).

$\mathrm{Na}$ dessensibilização há a intenção de colocar o paciente em estado de relaxamento, expondo gradualmente os procedimentos odontológicos, em um ambiente livre de distrações e simplificando a sala de atendimento. Já na distração introduz-se estímulos atrativos que desviam a atenção de elementos aversivos do consultório, que geram medo e tensão, para situações imaginárias agradáveis (Caldas Jr \& Machiavelli, 2013; Silva et al., 2016).

A ludoterapia entra com utilização do brinquedo para a transferência de medos, anseios e vontades, onde a criança consegue aprender por meio da brincadeira, de acordo com seu ritmo e capacidades. Essa técnica possibilita o aprendizado e consequentemente eleva a autoestima, diminuindo, assim, a ansiedade e possibilitando sua participação nas atividades e atendimento com maior motivação (Caldas Jr \& Machiavelli, 2013).

Em um estudo realizado com 12 pessoas com diagnóstico de SD pela Fundação CINDES de Pereira, com idades entre 8 e 49 anos, no ano de 2017, pôde-se evidenciar a eficácia da implementação de estratégias lúdicas como ferramenta fundamental para o alcance de uma aprendizagem significativa. Tendo como resultado essa população aprendendo a identificar as estruturas da cavidade bucal e os riscos de não realizar uma higiene bucal adequada, além de melhorar a execução da técnica de escovação e sua autonomia (Vargas-Ramírez et al., 2021).

Em último caso, e após esclarecimento para o responsável, tem-se a estabilização protetora, utilizada para um paciente não cooperativo que não se adaptou às outras formas de manejo comportamental e não pode cooperar devido seu estado emocional. Essa técnica tem como benefícios a redução ou eliminação dos movimentos indesejáveis e descontrolados, proteção do paciente, do dentista e do acompanhante. Entende-se como uma leve colocação da mão do auxiliar na criança, podendo se estender até o uso de acessórios para a contenção efetiva, como abridores de boca e "pacotes pediátricos" envolvendo a criança em imobilizadores específicos (Caldas Jr \& Machiavelli, 2013; Sant’anna et al., 2020).

O cuidado com a pessoa que vive com a síndrome de Down deve ser integral e multidisciplinar, sendo a presença do profissional da Odontologia muito importante no que se refere à motivação, supervisão e tratamento desses pacientes. É importante estar sempre em contato com os demais profissionais responsáveis pela criança, como médicos e psicólogos, buscando oferecer um tratamento inclusivo, de qualidade e pautado em todas as peculiaridades e adaptações necessárias que ele exige (Camera et al., 2011).

\section{Conclusão}

Verificou-se que ainda há controversas entre os autores quando se diz a respeito sobre o alto ou baixo índice de lesão de cárie em pacientes que vivem com a SD. Percebeu-se também que eles possuem características específicas extra e intra orais que podem interferir no manejo odontológico de rotina, sendo necessário direcionar e individualizar o tratamento. Salienta-se 
ainda a importância do acompanhamento familiar durante esse período, juntamente com instruções passadas pelo cirurgião dentista de como eles devem agir para facilitar o atendimento e passar mais confiança e conforto para a criança, bem como a necessidade de novos trabalhos acerca de métodos e abordagens mais específicos no atendimento desses pacientes.

Dessa forma, compreende-se que a atuação odontológica deve ser a mais precoce possível para que, desde a primeira infância, a criança acostume-se com o ambiente odontológico, com o profissional e até mesmo já crie hábitos que o sustentará para o resto da vida. Para isso, o cirurgião dentista necessita estar bem preparado para saber lidar com todas as características e particularidades de cada criança, entendendo seus medos, traumas, inseguranças e acima de tudo sua síndrome, que os deixam ainda mais especiais.

\section{Referências}

Albuquerque, C. M., Gouvêa, C. V. D., Moraes, R. C. M., Barros, R. N., \& Couto, C. F. do. (2010). Principais técnicas de controle do comportamento em Odontopediatria. Arq Cent Estud Curso Odontol Univ Fed Minas Gerais., 45 (2), 110-115. http://revodonto.bvsalud.org/pdf/aodo/v46n2/a08v46n2.pdf

Aljameel, A. H., Watt, R. G., Tsakos, G., \& Daly, B. (2020). Down syndrome and oral health: mothers' perception on their children's oral health and its impact. Journal of patient-reported outcomes, 4(1), 45. Doi: 10.1186/s41687-020-00211-y

Al-Maweri, S., \& Al-Sufyani, G. (2014). Dental caries and treatment needs of Yemeni children with down syndrome. Dental research journal, 11(6), 631-5. https://www.ncbi.nlm.nih.gov/pmc/articles/PMC4275630/pdf/DRJ-11-631.pdf

Brasil. Ministério da Saúde. (2020). "Não deixe ninguém para trás”: Dia Internacional da Síndrome de Down. https://bvsms.saude.gov.br/nao-deixe-ninguempara-tras-dia-internacional-da-sindrome-de-down-2020/

Brasil. Ministério da Saúde. Secretaria de Atenção Primária à Saúde. (2019). Guia de Atenção à Saúde Bucal da Pessoa com Deficiência. https://bvsms.saude.gov.br/bvs/publicacoes/guia_atencao_saude_bucal_pessoa_deficiencia.pdf

Brandenburg, O. J., \& Marinho-Casanova, M. L. (2013). A relação mãe-criança durante o atendimento odontológico: contribuições da análise do comportamento. Estudos de Psicologia, Campinas, 30(4), 629-640. https://www.scielo.br/j/estpsi/a/mdj6qQmVzCcNMkf9BG3f4tB/?lang=pt\&format=pdf

Caldas Jr, A. F., \& Machiavelli, J. L. (2013). Atenção e Cuidado da Saúde Bucal da Pessoa com Deficiência: protocolos, diretrizes e condutas para cirurgiõesdentistas. Editora: Universitária, Recife - UFPE, 1-232. https://cvtpcd.odonto.ufg.br/up/299/o/Livro_-_Eixo_2_-_Cirurgi\%C3\%B5esdentistas.pdf?1504016031

Camera, G. T., Mascarello, A. P., Bardini, D. R., Fracaro, G. B., \& Boleta-Ceranto, D. C. F. (2011). O papel do cirurgião-dentista na manutenção da saúde bucal de portadores de síndrome de down. Odontol. Clín.-Cient., 10 (3), 247-250. http://revodonto.bvsalud.org/pdf/occ/v10n3/a11v10n3.pdf

Cordeiro A. M., Oliveira G. M., Renteria J. M., Guimarães C. A., \& GERS-Rio (2007). Revisão sistemática: Uma revisão narrativa. Rev Col Bras Cir, 34(6). https://www.scielo.br/j/rcbc/a/CC6NRNtP3dKLgLPwcgmV6Gf/?lang=pt\&format=pdf.

Dias, M. R. F. M. (2013). Comunicação não-verbal no setting da consulta em odontopediatriainternational. Journal of Developmental and Educational Psychology, 1(1), 357-365. https://www.redalyc.org/articulo.oa?id=349852058028

Falcão, A. C. S. L. A., Santos, J. M., Nascimento, K. L. L., Santos, D. B. N., \& Costa, P. V. A. (2019). Síndrome de Down: abordagem odontopediátrica na fase oral. Rev. Odontol. Univ. Cid., São Paulo, 31(1), 57-67. https://docs.bvsalud.org/biblioref/2019/08/1009763/sindrome-de-down-abordagem-odontopediatricana-fase-oral.pdf

Gallo, C., Pastore, I., Beghetto, M., \& Mucignat-Caretta, C. (2019). Symmetry of dental agenesis in Down Syndrome children. Journal of dental sciences, 14(1), 61-65. Doi: https://doi.org/10.1016/j.jds.2018.04.003

Ghaith, B., Al Halabi, M., Khamis, A. H., \& Kowash, M. (2019). Oral Health Status among Children with Down Syndrome in Dubai, United Arab Emirates. Journal of International Society of Preventive \& Community Dentistry, 9(3), 232-239. https://doi.org/10.4103/jispcd.JISPCD_396_18

Khandelwal, M., Shetty, R. M., \& Rath, S. (2019). Effectiveness of Distraction Techniques in Managing Pediatric Dental Patients. Int J Clin Pediatr Dent, 12(1), 18-24. https://doi.org /10.5005/jp-journals-10005-1582

Morales-Chávez, M., Rada-Berroteran, A., \& Arcila-Ramos, L. (2014). Periodontal status of mentally handicapped school children in Caracas, Venezuela. A cross-sectional study. J Oral Res, 3(3): 156-161. https://pesquisa.bvsalud.org/portal/resource/pt/lil-730019

Moreira, M. J. S., Schwertner, C., Jardim, J. J., \& Hashizume, L. N. (2015). Cárie dentária em indivíduos com síndrome de Down: uma revisão sistemática. International Journal of Pediatric Dentistry, 26 (1), 3-12. Doi: https://doi.org/10.1111/ipd.12212

Moura, B. F., Imparato, J. C. P., Parisotto, T. M., \& Benedetto, M. (2015). Ansiedade infantil prévia à consulta odontológica: avaliação mediante instrumento lúdico como condicionamento. RGO, Porto Alegre, 63(4), 455-460. Doi: https://doi.org/10.1590/1981-863720150003000122848

Nacamura, C. A.,Yamashita, J. C., Busch, R. M. C., \& Marta, S. N. (2015). Síndrome de Down: inclusão no atendimento odontológico municipal. FOL (Faculdade de Odontologia de Lins/Unimep), 25(1) 27-35. https://www.metodista.br/revistas/revistas-unimep/index.php/Fol/article/view/2493/1530

Pini, D. M., Fröhlich, P. C., \& Rigo, L. (2016). Avaliação da saúde bucal em pessoas com necessidades especiais. Einstein, São Paulo, v.14(4), 501-507. Doi: https://doi.org/10.1590/S1679-45082016AO3712 
Research, Society and Development, v. 10, n. 14, e552101422602, 2021

(CC BY 4.0) | ISSN 2525-3409 | DOI: http://dx.doi.org/10.33448/rsd-v10i14.22602

Sant'anna, R. M. M.; Silva, R. A.; Silva, L. V.; Almeida, T.F. (2020). Aspectos éticos e legais das técnicas de manejo de comportamento em odontopediatria: uma revisão narrativa da literatura. Rev Bras Odontol Leg.,7(2),70-80. https://portalabol.com.br/rbol/index.php/RBOL/article/view/320/250

Santos, M. R. dos., Oliveira, K. L., Fonte, J. B. M. da., Anjos Hora, I. A. A., Takeshita, W. M., \& Melo, M. F. B. de. (2014). Prevalência de alterações dentárias em pacientes com síndrome de down avaliados por meio de radiografia panorâmica. Rev. Odontol. Univ. Cid. São Paulo, 26(2),112-8. https://arquivos.cruzeirodosuleducacional.edu.br/principal/old/revista_odontologia/pdf/maio_agosto_2014/Odonto_26_2014_112-118.pdf

Scalioni, F., Carrada, C., Machado, F., Devito, K., Ribeiro, L.C., Cesar, D., \& Ribeiro, R. (2017). Densidade salivar de Streptococcus mutans e Streptococcus sobrinus e cárie dentária em crianças e adolescentes com síndrome de Down. J. Appl. Oral Sci. 25 (3). Doi: https://doi.org/10.1590/1678-7757-2016-0241

Silva, L. F. P.,Freire, N. C., Santana, R. S., \& Miasato J. M. (2016). Técnicas de manejo comportamental não farmacológicas na Odontopediatria. Rev. Odontol. Univ. Cid. São Paulo, $28(2), 2$ https://arquivos.cruzeirodosuleducacional.edu.br/principal/old/revista_odontologia/pdf/maio_agisto_2016/Odonto_02_2016_135-142_1.pdf

Silva, A. F., \& Trabaquin, P. S. (2019). Assistência de enfermagem para crianças com sindrome de down. Revista da Saúde da AJES, 5(9), 1 -9. https://revista.ajes.edu.br/index.php/sajes/article/view/300

Usui, A., Campos, D. M., Shitsuka, C., Pedron I.G., \& Shitsuka, R. (2020). Características bucais e manejo comportamental de pacientes com Síndrome de Down. e-Acadêmica, 1(3), 15. Doi: https://doi.org/10.52076/eacad-v1i3.15

Vargas-Ramírez, J., Cardona-Cañas, M. F., Rivera-Suárez, M, F., Guerrero-Jaramillo, A. N., Duque-Mejía, M., Ospina-Metheus, P. A., \& García-Oyuela, A. F. (2021). Estrategias lúdicas para mejorar la higiene bucal en una población con síndrome de Down. Hacia la Promoción de la Salud., 26 (2), 23-37. Doi: https://doi.org/10.17151/hpsal.2021.26.2.3

Vilela, J. M. V., Nascimento, M. G., Nunes, J., \& Ribeiro, E. L. (2018). Características bucais e atuação do cirurgião-dentista no atendimento de pacientes portadores de sindrome de down. Caderno De Graduação - Ciências Biológicas E Da Saúde, 4(1), 89. https://periodicos.set.edu.br/facipesaude/article/view/6416 